\title{
Prospects for the Platinum Mode
}

\section{MASAKI ISHITSUKA*广}

Indiana University, Bloomington, Indiana 47405, USA

E-mail: ishitsuka@hep.phys.titech.ac.jp

The NOvA long baseline neutrino oscillation experiment will employ totally active scintillator detector. The detector is optimized to detect a few $\mathrm{GeV}$ electrons from $v_{e}$ appearance signal through sub-dominant $v_{\mu} \rightarrow v_{e}$ oscillations from a muon neutrino beam. Such detector technology with an additional function of magnetic field is also considered as a candidate of the detectors in Neutrino Factory to study $v_{\mu} \rightarrow v_{e}$ (and $\overline{v_{\mu}} \rightarrow \overline{v_{e}}$ ) oscillations. The performance of the NOvA detector design will be described in this paper.

10th International Workshop on Neutrino Factories, Super beams and Beta beams June 30 - July 52008

Valencia, Spain

\footnotetext{
*Speaker.

†Present address: Tokyo Institute of Technology, Tokyo, Japan
} 


\section{Introduction}

Since evidence for an atmospheric neutrino oscillations was reported by the Super-Kamiokande experiment in 1998 [1], significant improvements of the understanding of neutrino oscillation phenomenon and the measurement of oscillation parameters, that is mass squared difference and mixing angles, have been achieved by various experiments in last decade [2]. Measurement of the remaining unknown mixing angle $\theta_{13}$ may be realized by long baseline accelerator and reactor neutrino experiments in the next decade followed by a search for CP-violation in neutrino sector, which may give us a hint to understand the origin of the baryon asymmetry of our universe. Various experimental approaches to search for CP-violating phase $\delta$ and resolve mass ordering in neutrino sector have been studied for the wide range of $\theta_{13}$ value, which include long baseline neutrino oscillation experiments using high intensity muon neutrino beam, electron neutrino beam (beta-beam) and electron plus muon neutrino beam (Neutrino Factory). In a Neutrino factory, electron and muon neutrino beam are produced from muon decays: $\mu^{+} \rightarrow e^{+}+v_{e}+\overline{v_{\mu}}$ or $\mu^{-} \rightarrow e^{-}+\overline{v_{e}}+v_{\mu}$. This neutrino beam in a Neutrino Factory provides capability to study various neutrino oscillation modes, in which $v_{\mu} \rightarrow v_{e}$ oscillation ("platinum") channel is sensitive to CP-violating phase $\delta$ and mass ordering in neutrino sector. The detector to study this channel need to have capability to distinguish $v_{e}$ appearance signal from the background events due to $v_{\mu}$ charged-current (CC) and neutral-current (NC) interactions. In addition, further capability to distinguish electrons in $v_{e}$ $\mathrm{CC}$ interactions from positrons in $\overline{v_{e}} \mathrm{CC}$ interactions is required. One of the candidates of the detector which may satisfy those conditions is a totally active scintillator detector which will be also employed in the NOvA experiment, together with an additional function of magnetic field to achieve identification of electrons and positrons with the curvature of the tracks. The status and performance of the NOvA detector design [3] are discussed in the following sections.

\section{NOvA experiment}

The NuMI Off-axis Neutrino Appearance (NOvA) experiment is a next generation long baseline neutrino oscillation experiment which aims to search for non-zero $\theta_{13}$ in sub-dominant $v_{\mu} \rightarrow v_{e}$ oscillations approximately an order of magnitude beyond the current limit from the Chooz reactor neutrino experiment [4]. The NOvA experiment also has potential to search for CP-violation and resolve mass hierarchy in neutrino sector with the large matter effects induced by a $810 \mathrm{~km}$ long baseline. The NOvA experiment employs off-axis method using a muon neutrino beam created at the NuMI facility, by which an intense narrow band beam is available. The peak of the narrow band beam is put on the oscillation maximum at $2 \mathrm{GeV}$ to optimize the sensitivity to $v_{\mu} \rightarrow v_{e}$ oscillations. The NOvA experiment also employs two detectors method to reduce systematic errors on the measurement associated with the uncertainties on the neutrino flux prediction. Far and near detectors are based on the same technology, so that systematic uncertainties on the neutrino interaction cross-sections and detector modeling are largely canceled by comparing the measurements by two detectors. Figure 1 shows the expected energy spectra for $v_{e}$ appearance signal and the backgrounds assuming $\sin ^{2} 2 \theta_{13}=0.01, \sin ^{2} 2 \theta_{23}=1.0$ and $\Delta m_{23}^{2}=2.5 \times 10^{-3} \mathrm{eV}^{2}$ in the NOvA far detector. The detector is located at a distance of $810 \mathrm{~km}$ with $15 \mathrm{mrad}$ off-axis angle. 


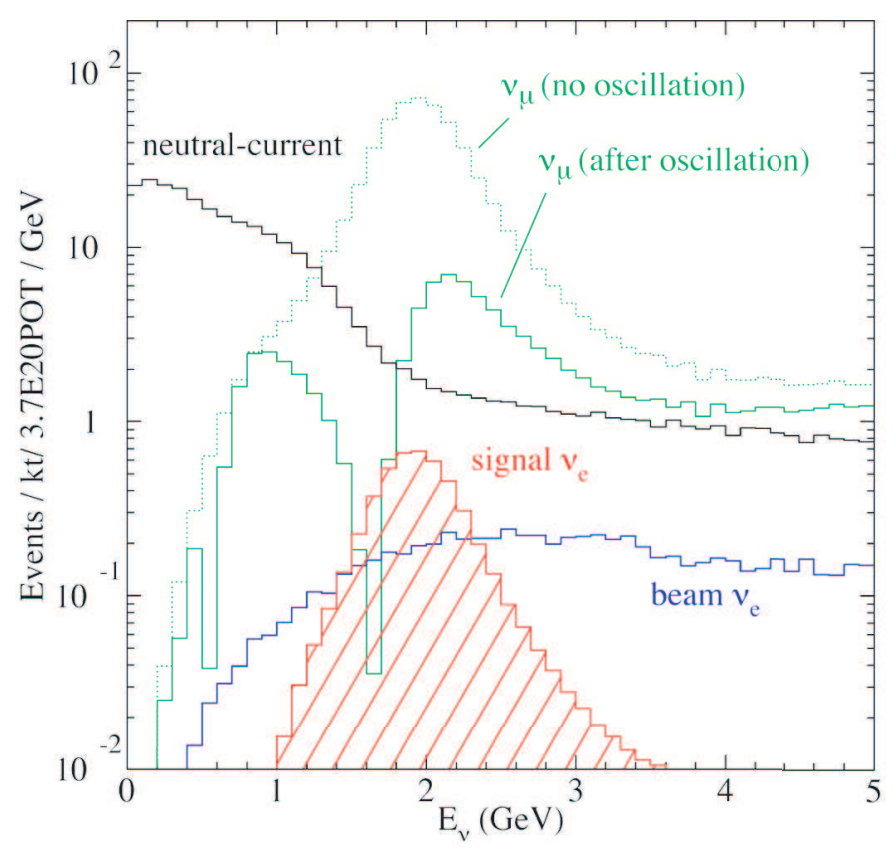

Figure 1: Simulated energy distributions for the $v_{e}$ oscillation signal, intrinsic $v_{e}$ in neutrino beam, NC events and $v_{\mu}$ CC events with and without neutrino oscillations assuming $\sin ^{2} 2 \theta_{13}=0.01, \sin ^{2} 2 \theta_{23}=1.0$ and $\Delta m_{23}^{2}=2.5 \times 10^{-3} \mathrm{eV}^{2}$. The detector is located at a distance of $810 \mathrm{~km}$ with $15 \mathrm{mrad}$ off-axis angle.

\section{NOvA detector}

The NOvA experiment will employ a totally active scintillator detector. The detector technology is designed to detect a few $\mathrm{GeV}$ electrons from $v_{e}$ appearance signal. The active detector medium is liquid scintillator made from mineral oil solvent mixed with pseudocumene scintillant and wavelength shifters. The liquid scintillator is filled in PVC extrusion cells with cross-section of $4 \mathrm{~cm}$ width and $6 \mathrm{~cm}$ thick. The length of the cells are $15.7 \mathrm{~m}$ at the far detector. The scintillator cells are arranged to form a plane, and the detector is made up by a series of planes. Scintillator cells in successive planes are rotated by $\pm 90^{\circ}$ to provide two orthogonal views of the track and reconstruct three-dimensional topology of particles by the combination. Photons emitted in the scintillator are transfered by wavelength shifting (WLS) fiber, and fed into Avalanche Photodiode (APD) where photons are converted to electric signal. Quantum efficiency of APD is $\sim 85 \%$ for those photons emitted by the WLS fiber and the gain is $\sim 100$. The temperature of APD is kept to be $-15^{\circ} \mathrm{C}$ during the operation to suppress the dark noise to $\sim 2$ p.e., in which signal over noise ratio is greater than 10 for muons at the far end in the far detector. Total mass of the far detector is 15 kton.

\section{Performance of the NOvA detector design}

Figure 2 shows the event displays for $v_{e} \mathrm{CC}, v_{\mu} \mathrm{CC}$ and NC interactions from the NOvA detector Monte Carlo simulation. Electron from $v_{e} \mathrm{CC}$ interaction forms electromagnetic shower in the detector, while clear straight line is created with the hit cells along the muon track in case 

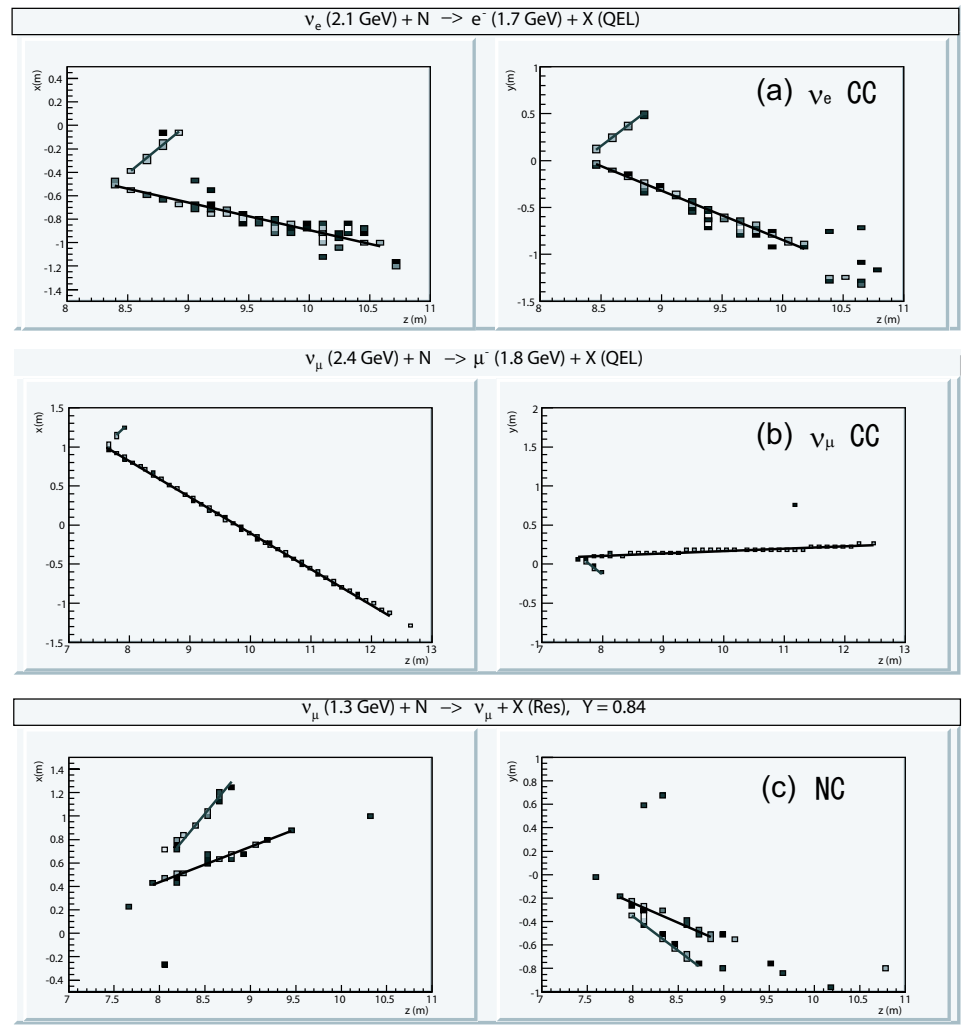

Figure 2: Event displays for (a) $v_{e} \mathrm{CC}$, (b) $v_{\mu} \mathrm{CC}$ and (c) $\mathrm{NC}$ with $0.9 \mathrm{GeV} \pi^{0}$ from NOvA Monte Carlo simulation. Left and rights figures show the horizontal and vertical planes, respectively. Neutrino beam comes from the left in each figure. Boxes represent hit cells and the overlaid lines indicate the reconstructed tracks.

of $v_{\mu} \mathrm{CC}$ interaction. Short proton track is also visible in the $v_{\mu} \mathrm{CC}$ interaction. Proton track identification is expected in the NOvA detector using the large $d E / d x$ at the end of the track. Typical NC interactions leave diffused hits and only short tracks are reconstructed. NC interactions often produce $\pi^{0}$ which decays to two $\gamma^{\prime}$ s. Those are the major source of the backgrounds to $v_{e}$ $\mathrm{CC}$ interactions, though most of them are distinguished by the event topology. $v_{\tau}$ from $v_{\mu} \rightarrow v_{\tau}$ oscillations are mostly at around $2 \mathrm{GeV}$ which is well below the threshold of $v_{\tau} \mathrm{CC}$ interactions at $3.5 \mathrm{GeV}$, and is not observable in the NOvA detector.

Performance of the NOvA detector design to separate $v_{e}$ appearance signal and backgrounds was studied using the Monte Carlo simulation based on the measurements of the characteristics for the liquid scintillator, WLS fiber, APD and front-end electronics. Selection of $v_{e}$ appearance signal is carried out using an algorithm based on a multi-variables artificial neural network method as the inputs from the NOvA event reconstruction, which includes a $\chi^{2}$ from a fit to the scintillator hit distributions assuming an electromagnetic shower profile and the transverse size of the shower. Efficiency for the $v_{e}$ appearance signal was obtained as $36 \%$ including the fiducial volume cut, while the remaining backgrounds are less than $0.1 \%$ of $v_{\mu}$ CC interactions, $0.23 \%$ of NC interactions and $14 \%$ of intrinsic $v_{e}$ in neutrino beam. It is basically impossible to distinguish individual $v_{e}$ CC interaction due to intrinsic $v_{e}$ in the neutrino beam from $v_{e}$ appearance signal, on the other 
hand the beam $n u_{e}$ has broad distribution while $v_{e}$ appearance signal peaks at around $2 \mathrm{GeV}$. Therefore, the beam $v_{e}$ backgrounds are reduced by requiring the energy in the signal region. Assuming $\sin ^{2} 2 \theta_{13}=0.10, \sin ^{2} 2 \theta_{23}=1.0, \Delta m_{23}=2.4 \times 10^{-3} \mathrm{eV}^{2}$ and no matter effects for 3 years of neutrino (anti-neutrino) running at $700 \mathrm{~kW}$ beam power $\left(6 \times 10^{20} \mathrm{pot} / \mathrm{year}\right)$, the expected $v_{e}$ signal events after selection is 75.0 (29.0), while total backgrounds are 14.4 (7.6) events. The NC and intrinsic $v_{e}$ events are comparable contributions to the backgrounds, while $v_{\mu} \mathrm{CC}$ backgrounds are negligible compared to them.

\section{Summary}

Totally active scintillator detector is considered as one of the candidate detectors to study $v_{\mu} \rightarrow v_{e}$ oscillation channel in a Neutrino Factory. The NOvA experiment studied the performance of the $v_{e}$ appearance signal separation against the backgrounds which are mainly due to NC interactions. The sensitivity to search for non-zero $\theta_{13}$ was approximately an order of magnitude beyond the current limit. Additional function of the magnetic field is required in the use of this detector technology in Neutrino Factory in order to separate electrons from $v_{\mu} \rightarrow v_{e}$ oscillations from positrons in neutrino beam (and vise-versa in $\mu^{+}$running).

\section{References}

[1] Y. Fukuda et al, Phys. Rev. Lett. 811562 (1998).

[2] W.-M. Yao et al, J. Phys. G33 1 (2006).

[3] D. Ayres et al, “The NOvA Technical Design Report”, Fermilab-design-2007-01 (2007)

[4] M. Apollonio et al, Eur. Phys. J. C27 331 (2003). 